\title{
Effects of long-term home-based Liuzijue exercise combined with clinical guidance in elderly patients with chronic obstructive pulmonary disease
}

This article was published in the following Dove Press journal:

Clinical Interventions in Aging

\author{
Peijun $\mathrm{Li}^{1, *}$ \\ Jingxin Liul,* \\ Yufan Lu' \\ Xiaodan Liu \\ Zhenwei Wang ${ }^{3}$ \\ Weibing $\mathrm{Wu}^{\prime}$ \\ 'Department of Sports Medicine, \\ Shanghai University of Sport, Shanghai, \\ People's Republic of China; ${ }^{2}$ School \\ of Rehabilitation Medicine, Shanghai \\ University of Traditional Chinese \\ Medicine, Shanghai, People's Republic \\ of China; ${ }^{3}$ Department of Respiratory \\ Medicine, Yueyang Hospital of \\ Integrated Traditional Chinese and \\ Western Medicine, Shanghai, People's \\ Republic of China \\ *These authors contributed equally \\ to this work
}

Correspondence: Weibing Wu

Department of Sports Medicine, Shanghai University of Sport, Heng Ren Road No I88, Yang Pu District, Shanghai 200438, People's Republic of China

Tel +862151253319

Fax +86 2I 51253380

Emailwwb75@I26.com
Purpose: This study was designed to investigate the effects of long-term home-based Liuzijue exercise combined with clinical guidance in elderly patients with chronic obstructive pulmonary disease (COPD).

Methods: Forty patients with COPD at stages II-III of the Global Initiative for Chronic Obstructive Lung Disease were enrolled. The subjects were randomly allocated to the Liuzijue exercise group (LG) or control group (CG) in a 1:1 ratio. Participants in the LG performed six Liuzijue training sessions, including 4 days at home and 2 days in the hospital with clinical guidance for 60 minutes/day for 6 months. Participants in the CG conducted no exercise intervention. In addition, lung function test, 6-minute walking test (6MWT), 30-second sit-to-stand test (30 s SST), and the St George's Respiratory Questionnaire (SGRQ) were conducted at the baseline and at the end of the intervention.

Results: Thirty-six patients completed the study. The patients' lung function improved significantly $(p<0.05)$ in the LG as well as the 6MWT, 30 s SST, and SGRQ score $(p<0.01)$. While the SGRQ total score, activity, and impact scores increased significantly $(p<0.05)$ in the CG. In addition, there were significant differences between the groups $(p<0.01)$ in regard to the values of forced expiratory volume in 1 second as a percentage of the predicted volume, 6MWT, $30 \mathrm{~s} \mathrm{SST}$, and SGRQ.

Conclusions: Long-term home-based Liuzijue exercise combined with clinical guidance can effectively improve the pulmonary function, exercise capacity, and quality of life of elderly patients with moderate to severe COPD.

Keywords: chronic obstructive pulmonary disease, elderly patients, Liuzijue exercise, pulmonary function, exercise capacity, quality of life

\section{Introduction}

Chronic obstructive pulmonary disease (COPD) is a preventable and treatable disease that is characterized by persistent respiratory symptoms and airflow obstruction. ${ }^{1}$ While the mortality rate of COPD gradually increased from 2005 to 2015 due to global aging, the overall age-specific mortality rate decreased. ${ }^{2}$ These trends suggest that the mortality rate of COPD has not been effectively alleviated. Effective interventions should be urgently developed.

Exercise training is an important component of pulmonary rehabilitation, as recommended to patients with COPD by the American Thoracic Society/European Respiratory Society (ATS/ERS). ${ }^{3}$ Furthermore, exercise training can ameliorate the decline in lung function, improve exercise capacity, reduce the number of acute attacks, reduce rehospitalization and mortality, and improve the quality of life of COPD patients..$^{4,5}$ Although 
high-intensity exercises are more effective in relieving dyspnea and increasing patient exercise capacity than low-intensity exercises, ${ }^{6} \mathrm{COPD}$ patients with dyspnea and exercise intolerance may not be able to endure high-intensity exercises. ${ }^{7}$ In addition, given the monotonous exercise mode and the need for special equipment and locations, conventional exercises may decrease the compliance of patients. Therefore, a convenient and effective exercise mode is needed to satisfy the demands of COPD patients to rehabilitation.

Traditional Chinese fitness exercises, such as Tai Chi, five-animal exercises, and Liuzijue, ${ }^{8,9}$ are low-moderateintensity exercises that are easy to learn and not limited by location or the need for special equipment. Moreover, these exercises are appreciated by the elderly and previous studies found that traditional Chinese fitness exercise can significantly improve the balance and exercise capacity of healthy people ${ }^{10}$ and exert positive effects on patients with chronic diseases. ${ }^{11,12}$ Liuzijue, featuring the combined respiratory pattern of abdominal breathing and pursed lip breathing, is performed by expiration to producing six different sounds (xu, he, hu, si, chui, and xi) together with the corresponding body movements. ${ }^{9}$ This type of respiratory pattern can retard the expiratory flow rate and modify rapid shallow breathing patterns, thereby helping COPD patients maintain proper airway pressure, prevent premature tracheal closure, and improve gas exchange. ${ }^{13-15}$ In addition, upper and lower limb exercises can promote the function of the accessory respiratory muscles, refine the coordination of the upper and lower limbs, and improve neuromuscular control, which further improve the exercise capacity of patients. ${ }^{9}$

Previous studies found that exercise interventions for pulmonary function had different results (improved or not), ${ }^{16-19}$ and the inconsistent results may be attributed to a range of factors, including the variations in patients' health conditions, the extent of provided clinical supervision, and the amount of time dedicated to the intervention. However, different exercise programs for functional exercise capacity and quality of life of patients with COPD have consistent improvement results. In addition, previous studies have mostly focused on single clinic- or home-based interventions, while supervised clinic-based rehabilitation results in a considerable family and social burden. Consequently, an effective home-based rehabilitation program should be explored.

This research mainly aimed to 1) investigate the effects of long-term homed-based Liuzijue exercise combined with clinical guidance on the lung function, exercise capacity, and quality of life of elderly patients with COPD and the patients' compliance with intervention through a randomized controlled trial and 2) determine an effective and affordable rehabilitation program for elderly patients with COPD. The present study hypothesized that a 6-month home-based Liuzijue exercise combined with clinical guidance can significantly improve the pulmonary function, exercise capacity, and quality of life of patients with stable COPD.

\section{Methods \\ Study patients}

Patients with COPD were recruited from Yue-Yang Integrative Medicine Hospital (which is affiliated with the Shanghai University of Traditional Chinese Medicine) from March 2013 to March 2014.

Patients were enrolled if they satisfied the inclusion criteria as follows: diagnosis of COPD in accordance with the 2013 Global Initiative for Chronic Obstructive Lung Disease (GOLD) criteria; ${ }^{20}$ gender was not limited but the patients had to be between 40 and 80 years of age; forced expiratory volume in 1 second (FEV 1)/forced vital capacity $(\mathrm{FVC})<0.7$ after treatment with a bronchodilator and $30 \% \leq$ FEV $1 \%$ pred $<80 \%$; patients had not attended a regular exercise program for at least 6 months prior to enrollment; patients were stable for the 4 weeks prior to randomization; and they had voluntarily signed an informed consent document.

Participants were excluded if they satisfied the following criteria: they had an acute exacerbation of COPD; participants were younger than 40 or older than 80 ; they had bronchiectasis, bronchial asthma, bronchial tumor, tuberculosis, or cancer; they had severe cardiovascular, cerebrovascular, liver, kidney, or hematopoietic system disease, or a mental disorder; women who were pregnant or lactating; and patients who were extremely weak or had a physical disability.

\section{Study design}

This work is a single-blind, randomized controlled trial, which included random allocation, allocation concealment, and the blinding of the outcome evaluators. The enrolled participants were randomly assigned in a 1:1 ratio to the control group (CG) or the Liuzijue exercise group (LG) by computer-generated randomized numbers. The randomization and distribution were conducted by a person who was not involved in the recruitment of the patients. Outcome assessors were blinded to the group allocation. All the subjects continued their prescribed medical treatments. Outcome measures were conducted within 1 week at the baseline and after 6 months of intervention. The study was approved by the Ethics Committee of Yue-Yang Integrative 
Medicine Hospital (affiliated with the Shanghai University of Traditional Chinese Medicine). All the participants signed the informed consent.

\section{Intervention}

Patients in the CG accepted the usual care provided according to the GOLD guidelines, including pharmacologic therapy, smoking cessation, and education. Patients in the CG did not participate in any exercise interventions. Based on the usual care, patients in the LG conducted Liuzijue exercise according to the Health Qigong Liuzijue program, which was compiled by the Chinese Health Qigong Association. ${ }^{21}$

During the first 2 weeks after allocation, patients in the LG studied and practiced Liuzijue exercise three times a week. Meanwhile, videos of the Health Qigong Liuzijue and an exercise log were provided to the patients in LG. Subsequently, patients performed the Liuzijue exercise four times a week at home and twice a week at the hospital under the guidance of a specialist. The extent of the clinic exercise supervision was based on a previous study. ${ }^{3}$ In the present study, the main purpose of the clinical exercise supervision was to guarantee the patients' safety and their correctness in executing the exercise program as well as to motivate the patients to engage in the exercise. The entire training process was also recorded including the training duration, training intensity, and the training site.

The program of LG included the following: 1) warm-up: patients performed joint activities under the guidance of an instructor for 5-10 minutes; 2) Liuzijue exercise: patients performed intact Liuzijue exercise, which included $\mathrm{xu}$, he, $\mathrm{hu}, \mathrm{si}$, chui, xi and the corresponding actions with an instructor for 40 minutes; 3) cooling-down: patients adjusted their breathing and strengthening of muscles for 5-10 minutes. The exercise program was conducted twice a week under the leadership of a specialist at the Yue-Yang Integrative Medicine Hospital of the Shanghai University of Traditional Chinese Medicine from 9:00-10:00 am and four times a week at home or in a nearby park for $60 \mathrm{~min}$ based on the patients' time for a total of six times a week for 6 months.

The exercise intensity was set at a score of 3-4 on the Borg 0-10 scale. ${ }^{22}$ While the exercise intensity was lower than the pre-set target exercise intensity, patients were encouraged to adjust the motion and posture or the degree of the extensions to improve their exercise intensity.

\section{Adherence}

For patients in the $\mathrm{CG}$, we quantified the adherence through the number of responses to the telephone calls placed once a week by a specialist. Patients were classified as compliant if the completion of responses to the telephone calls was no less than $85 \%$.

To monitor the LG patients' adherence during the intervention, we quantified their adherence through the activities in the exercise log registered by patients, attendance at the supervised training sessions in the hospital, and the number of responses to the telephone calls placed once a week by the specialist. Patients were classified as compliant if the completion of exercise $\log$ and responses to the telephone calls were no less than $85 \%$.

\section{Outcome measures}

Outcome measures were determined at the baseline (week 0) and after 6 months of the intervention. These measures included the basic characteristics of the patients, pulmonary function, 6-minute walking distance (6MWD), 30-second sitto-stand test (30 SST), and quality of life. All these data were measured within 7 days after the end of the intervention.

\section{Pulmonary function tests}

Pulmonary function was assessed by a spirometer (MSDiffusion, Jaeger, Vyaire Medical, Lake Forest, IL, USA) that was operated by a physician in the Respiratory Department of Lung Function in Yue-Yang Hospital according to the recommendations of ATS/ERS. ${ }^{23}$ During the test, the patients were instructed to breathe three times. The best value was used for the analysis. The index used in this study was FEV 1\%pred and FEV 1/FVC after salbutamol inhalation for 15 minutes.

\section{6-minute walking test (6MWT)}

The 6MWT was performed with patients walking back and forth along a straight and flat corridor for 30 meters at the Rehabilitation Department of Yue-Yang Hospital in accordance with the recommendations of the ATS. ${ }^{24}$ Patients determined their own walking velocity. The test was conducted without assistance and using standard phrases of encouragement at the end of each minute. Discomfort and walking distance with an accuracy of 0.5 meter were recorded. In addition, a respiratory physician was present during the test to observe and record the discomfort events and levels of the patients.

\section{0 s SST}

According to the procedures provided in the study from Ozalevli et al, ${ }^{25}$ the $30 \mathrm{~s}$ SST was performed using an armless 
straight back chair, which was $43 \mathrm{~cm}$ high and placed against the wall. Participants were seated with their arms folded across their chest, their back firmly against the seat rest, and their feet placed flat on the floor to prepare for the test. Before the formal test, patients were instructed and familiarized with the test once or twice. Patients were asked to complete the sit-to-stand motions as many times as possible within 30 seconds.

\section{Quality of life}

Health-related quality of life was assessed using the St George's Respiratory Questionnaire (SGRQ), which is composed of 50 items divided into three domains (symptom, activity, and impact). Patients were asked to complete the questionnaire independently at a prescribed time. The SGRQ score was calculated by the software developed by the Peking Union Medical College Respiratory Medicine Development (Chinese version). The total scores of the SGRQ ranged from 0-100, with a high score indicating poor health.

\section{Statistical analysis}

Data obtained in this study were analyzed using SPSS version 23.0 (IBM Corporation, Armonk, NY, USA). Continuous variables are presented as mean \pm standard deviation (SD) unless otherwise stated. The data were tested for normal distribution using the Kolmogorov-Smirnov test, and the variance homogeneity was tested with the $F$-test. The baseline differences and the adherence rate differences between groups were compared using the independent $t$-test, and the differences within group was compared using the paired $t$-test. Changes from the baseline to the end of the study were calculated and expressed as mean \pm standard error of the mean. Based on general linear models, the analysis of covariance models was used to analyze the differences between groups after intervention. Categorical data are described as frequencies (in percentage). The chi-square test was used to analyze the intergroup differences. A $p$-value $<0.05$ was defined as statistically significant.

\section{Results}

Forty patients were enrolled, while thirty-six of these patients (90\%) completed the trial, with 19 and 17 patients in the CG and LG, respectively (Figure 1). The adherence rate of the LG patients was $88 \%$ (15 of the 17 patients with completed rate no less than $85 \%$ ), while the adherence rate of the $\mathrm{CG}$ patients was $95 \%$ (18 of 19 patients with completed rate no less than $85 \%$ ). There was no statistical significance between the groups $(p>0.05)$. According to the results of the statistical analysis, no significant difference was observed between the groups at baseline, which indicated good comparability (Table 1). Similarly, no differences were observed in the baseline lung function, 6MWD, $30 \mathrm{~s} \mathrm{SST}$, and SGRQ scores between the two groups $(p>0.05)$.

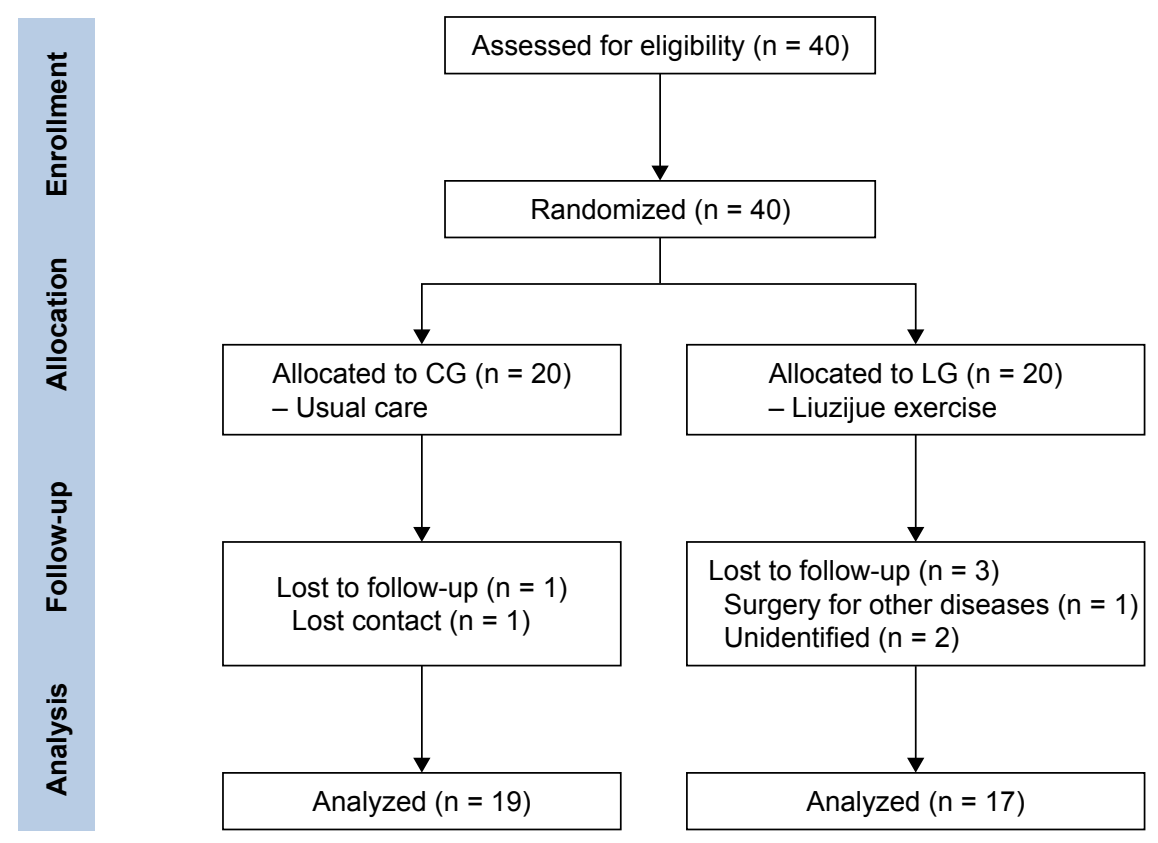

Figure I Study flow diagram.

Abbreviations: CG, control group; LG, Liuzijue exercise group. 
Table I Baseline characteristics of participants

\begin{tabular}{|c|c|c|c|}
\hline Characteristics & CG $(n=19)$ & LG $(n=17)$ & p-value* \\
\hline Age (years) & $66 \pm 9$ & $66 \pm 9$ & 0.98 \\
\hline Gender (male/female, $\mathrm{n}$ ) & $14 / 5$ & $14 / 3$ & 0.69 \\
\hline Years of COPD & $9 \pm 4$ & $8 \pm 5$ & 0.57 \\
\hline $\mathrm{BMI}, \mathrm{kg} / \mathrm{m}^{2}$ & $23 \pm 4$ & $23 \pm 4$ & 0.92 \\
\hline Smoking history (yes/no, n) & $16 / 3$ & $14 / 3$ & $\mathrm{I}$ \\
\hline Smoking status, n (\%) & & & 0.35 \\
\hline Current smoker & $6(31.6 \%)$ & $2(11.8 \%)$ & \\
\hline Ex-smoker & $10(52.6 \%)$ & $12(70.6 \%)$ & \\
\hline Smoking consumption, $\mathrm{n}(\%)$ & & & 0.87 \\
\hline$<15$ packs/year & $2(10.5 \%)$ & I (5.9\%) & \\
\hline 15-29 packs/year & $3(15.8 \%)$ & $3(17.6 \%)$ & \\
\hline$\geq 30$ packs/year & $14(73.7 \%)$ & $13(76.5 \%)$ & \\
\hline COPD stage, n (\%) & & & 0.73 \\
\hline Grade II & $13(68.6 \%)$ & $10(58.8 \%)$ & \\
\hline Grade III & $6(31.4 \%)$ & 7 (4I.2\%) & \\
\hline
\end{tabular}

Notes: Data are expressed as mean \pm standard deviation or $\mathrm{n}(\%)$. Independent sample $t$-test was used to compare age, years of COPD, and BMI between groups. Chi-square test was used to compare gender, smoking history, smoking status, smoking consumption, and COPD stage between groups. The significance level was set at $p<0.05$. * $p$-value for the comparison of outcome variables between groups. Abbreviations: BMI, body mass index; CG, control group; COPD, chronic obstructive pulmonary disease; LG, Liuzijue exercise group.

After 6 months of the intervention, the FEV 1\%pred and FEV $1 / \mathrm{FVC} \%$ improved significantly in the patients of the LG ( $p=0.006$ and $p=0.037$, respectively). While patients in the CG showed a decreasing trend in these values (Table 2 and Figure 2). Referring to exercise capacity, the 6MWD and $30 \mathrm{~s}$ SST values were significantly improved in the patients of LG ( $p=0.001)$, whereas the patients in the CG showed no change after intervention (Table 2 and Figure 3 ). Moreover, the SGRQ score in patients of the LG decreased significantly $(p<0.01)$. The impact and activity score in the patients of the CG increased significantly ( $p=0.043$ and $p=0.003$, respectively) (Table 3 ). Intergroup comparisons showed that the improvement in the FEV 1\%pred, 6MWD, 30s SST, and SGRQ scores in the patients of the LG was significantly different compared with that in the CG $(p<0.01)$ (Tables 2 and 3$)$.

\section{Effect-size values}

The 6MWD, as a sensitive index to reflect exercise tolerance, is closely related to the clinical conditions of patients. Consequently, we used the 6MWD as the primary outcome measure in this study. With $\alpha=0.05$ as a statistically significant difference, and a known sample size, the effectsize values were calculated. A $\beta=0.41$ and power $=1-\beta$ were obtained. Statistics presuming power $\geq 0.5$ indicated the modest validity. Thus, in this study, the Liuzijue exercise exerted a moderate treatment effect on the improvement of the 6MWD in patients with COPD.

\section{Discussion}

Liuzijue exercise is a traditional Chinese fitness exercise that is easy to learn and free from special location and equipment; since the exercise does not require anything other than a practitioner, it can reduce the costs associated with maintaining health and reduce the burden on a COPD sufferer's family. Results provide clinical support for encouraging elderly patients with stable COPD to practice home-based Liuzijue exercise combined with clinical guidance, which can significantly improve the pulmonary function, $6 \mathrm{MWD}$, $30 \mathrm{~s}$ SST, and quality of life. In addition, no adverse effects after 6-month intervention were found based on physician observations and participant feedback.

In the present study, the adherence rate over a 6-month Liuzijue exercise intervention was $88 \%$, and it was relatively higher than previous studies stated as follow. A previous study which conducted a 3-month community-based progressive training program had an adherence rate of $83 \%,{ }^{26}$ and another study using a 3-year pulmonary rehabilitation maintenance program had an adherence rate of $66 \% .{ }^{27}$ The inconsistent adherence rate may be attributed to the patients' condition and duration of intervention program. A low adherence rate has been related to lower exercise capacity, ${ }^{27}$ and a progressive training program may be difficult for many

Table 2 Comparison of changes in pulmonary function and exercise capacity

\begin{tabular}{|c|c|c|c|c|c|c|c|c|c|}
\hline \multirow[t]{2}{*}{ Parameters } & \multicolumn{4}{|l|}{ CG (n = 19) } & \multicolumn{4}{|l|}{ LG $(n=17)$} & \multirow[t]{2}{*}{$p^{c}$} \\
\hline & Pre & Post & $p^{a}$ & Changes & Pre & Post & $p^{b}$ & Changes & \\
\hline FEV I\%pred, \% & $58.49 \pm 19.4$ & $57.13 \pm 18.3$ & 0.09 & $-1.36 \pm 0.78$ & $55.50 \pm 16.8$ & $63.06 \pm 21.6$ & 0.006 & $7.57 \pm 2.42$ & 0.001 \\
\hline FEV I/FVC\%, \% & $60.78 \pm 10.3$ & $58.07 \pm 13.0$ & 0.47 & $-2.7 I \pm 3.65$ & $56.93 \pm 12.8$ & $62.28 \pm 16.4$ & 0.037 & $5.36 \pm 2.36$ & 0.136 \\
\hline 6MWD, meters & $417.47 \pm 64.05$ & $418.71 \pm 66.19$ & 0.77 & $1.24 \pm 4.14$ & $435.41 \pm 64.18$ & $483.62 \pm 67.30$ & 0.001 & $48.21 \pm 11.45$ & $<0.001$ \\
\hline $30 \mathrm{~s}$ SST, repetitions & $16.26 \pm 3.03$ & $16.58 \pm 3.15$ & 0.27 & $0.32 \pm 0.28$ & $16.65 \pm 2.96$ & $19.06 \pm 3.82$ & 0.001 & $2.4 I \pm 0.56$ & 0.002 \\
\hline
\end{tabular}

Notes: Pre and post data are expressed as mean \pm standard deviation. Changes were calculated by post minus pre and expressed as mean \pm standard error of the mean. The paired $t$-test was used to analyze within-group difference, and the analysis of covariance model was used to analyze the differences between groups after intervention. $p^{a}$ values for comparison of outcome variables from baseline to 6 months within control group. $p^{\mathrm{b}}$ values for comparison of outcome variables from baseline to 6 months within Liuzijue exercise group. $p^{c}$ values for the comparison of outcome variables from baseline to 6 months between groups.

Abbreviations: 6MWD, 6-minute walking distance; 30 s SST, 30-second sit-to-stand test; CG, control group; FEV I, forced expiratory volume in I second; FVC, forced vital capacity; LG, Liuzijue exercise group. 


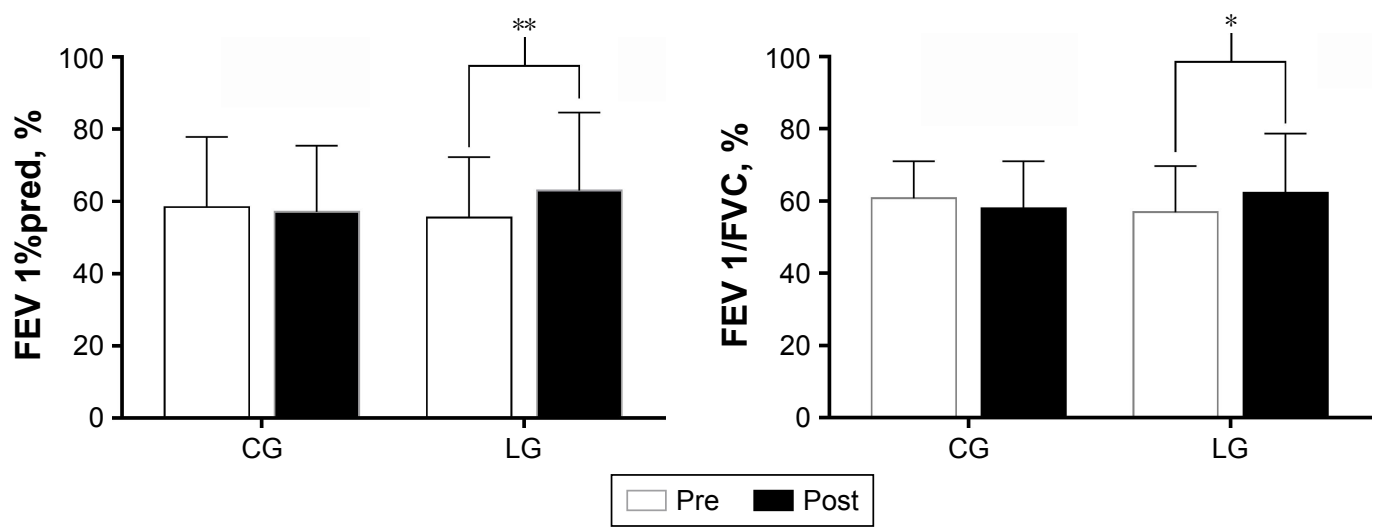

Figure 2 Pulmonary function in the CG and LG groups after 6-month training period.

Notes: ${ }^{*} p<0.05$, comparisons were significant within groups; ${ }^{* *} p<0.01$, comparisons were significant within groups.

Abbreviations: CG, control group; FEV I\%pred, forced expiratory volume in I second as a percentage of predicted; FEV I, forced expiratory volume in I second; FVC, forced vital capacity; LG, Liuzijue exercise group.

patients. The high adherence rate in this study suggests that this program of long-term home-based Liuzijue exercise combined with clinical guidance was well accepted by the participating patients.

Both long-term regular lung rehabilitation and short-term rehabilitation ( 8 weeks) can ameliorate lung function in patients with stable COPD. ${ }^{27,28}$ In contrast, other studies found no significant improvement in lung function after intervening in patients who had an acute exacerbation of COPD. ${ }^{29}$ Also, the program of home-based pulmonary rehabilitation has also shown no improvements in lung function. ${ }^{30}$ The discrepancy between these results and ours may be attributed to different disease conditions of the patients and extent of clinical supervision in the rehabilitation program. However, traditional Chinese fitness exercises can significantly improve the pulmonary function of patients with stable COPD, ${ }^{16}$ such as an intervention program that included 4 days of exercise at home and 3 days of exercise at the hospital. ${ }^{17}$ The results suggested that long-term home-based traditional Chinese fitness exercises combined with clinical guidance can potentially improve lung function in patients with COPD. Like previous studies, this present study applied a program of 4 days of home-based exercise and 2 days of clinic-based Liuzijue exercise which showed that the patients' lung function was significantly improved after 6 months of the intervention, and the improvement of FEV 1\%pred was significantly different between the groups. These results suggest that a program of long-term home-based Liuzijue exercise combined with clinical guidance can improve pulmonary function, although the optimal extent of supervision in the exercise intervention program is unclear.

Due to their respiratory symptoms, patients with COPD often complain about their exercise intolerance and decreased exercise capacity. Aerobic exercise training, as an essential component of pulmonary rehabilitation, can effectively enhance the exercise capacity of patients with COPD. Both high-intensity interval aerobic training and low-intensity
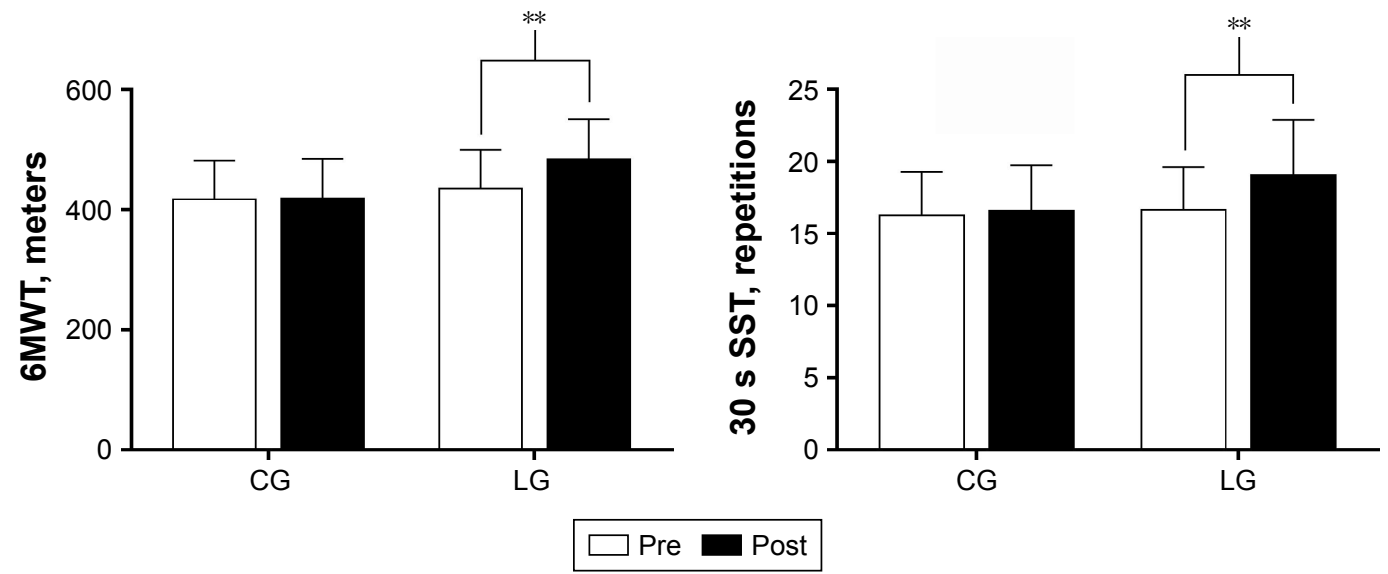

Figure 3 Exercise capacity in the CG and LG groups after 6-month training period.

Note: ${ }^{* *} p<0.01$, comparisons were significant within groups.

Abbreviations: 6MWT, 6-minute walking test; 30 s SST, 30-second sit-to-stand test; CG, control group; LG, Liuzijue exercise group. 
Table 3 Comparison of quality of life assessed by SGRQ

\begin{tabular}{|c|c|c|c|c|c|c|c|c|c|}
\hline \multirow[t]{2}{*}{ Parameters } & \multicolumn{4}{|l|}{ CG $(n=19)$} & \multicolumn{4}{|l|}{ LG $(n=17)$} & \multirow[t]{2}{*}{$P^{c}$} \\
\hline & Pre & Post & $p^{a}$ & Changes & Pre & Post & $p^{b}$ & Changes & \\
\hline Dyspnea score & $52.79 \pm 18.54$ & $56.63 \pm 21.61$ & 0.199 & $3.84 \pm 2.88$ & $58.71 \pm 17.95$ & $32.53 \pm 17.32$ & $<0.001$ & $-26.18 \pm 4.33$ & $<0.001$ \\
\hline Impact score & $29.21 \pm 14.91$ & $35.16 \pm 18.67$ & 0.043 & $5.95 \pm 2.8$ & $30.35 \pm 19.96$ & $17.12 \pm 13.58$ & $<0.001$ & $-13.24 \pm 2.99$ & $<0.001$ \\
\hline Activity score & $43.32 \pm 17.00$ & $51.63 \pm 23.42$ & 0.003 & $8.32 \pm 2.46$ & $46.06 \pm 19.03$ & $29.94 \pm 13.78$ & $<0.001$ & $-16.12 \pm 3.64$ & $<0.001$ \\
\hline Total score & $37.37 \pm 15.13$ & $45.78 \pm 16.36$ & 0.006 & $6.58 \pm 2.14$ & $39.88 \pm 17.26$ & $23.71 \pm 11.47$ & $<0.00 \mathrm{I}$ & $-16.18 \pm 2.4$ & $<0.001$ \\
\hline
\end{tabular}

Notes: Pre and post data are expressed as mean \pm standard deviation. Changes were calculated by post minus pre and expressed as mean \pm standard error of the mean. The paired $t$-test was used to analyze within-group difference, and the analysis of covariance model was used to analyze the differences between groups after intervention. $p^{\mathrm{a}}$ values for comparison of outcome variables from baseline to 6 months within control group. $p^{\mathrm{b}}$ values for comparison of outcome variables from baseline to 6 months within Liuzijue exercise group. $p^{c}$ values for comparison of outcome variables from baseline to 6 months between groups.

Abbreviations: CG, control group; LG, Liuzijue exercise group; SGRQ, St George's Respiratory Questionnaire.

aerobic training via traditional Chinese fitness exercise can effectively improve the 6MWD in COPD patients. ${ }^{17-19,31}$ Furthermore, a meta-analysis showed that the effects of aerobic exercise combined with resistance exercise in improving 6MWD are similar to those of aerobic exercise alone. ${ }^{32}$ The mentioned results suggest that the exercise capacity in patients with COPD can be effectively improved by aerobic exercise, even at a low intensity. Consistent with previous studies, our study showed that the 6MWD was significantly increased $(48.21 \mathrm{~m})$ and exceeded the minimum clinically important difference of 30 meters. $^{33}$

In addition, the present study applied the $30 \mathrm{~s}$ SST to reflect the lower extremity muscle strength and function, balancing ability, and transfer skills. Previous study found that the $30 \mathrm{~s}$ SST may be more sensitive than the 6MWT when evaluating the functional exercise capacity in patients with severe COPD because the hemodynamic stress at $30 \mathrm{~s}$ SST is less than that at 6MWT, whereas the two tests display a similar effect when assessing the exercise capacity. ${ }^{25}$ In addition, the result of the five times sit-to-stand test (FTSST) is moderately associated with mortality in patients with COPD. ${ }^{34}$ The results of $30 \mathrm{~s} \mathrm{SST}$ are significantly related to age, quality of life, dyspnea, and peripheral skeletal muscle strength. ${ }^{35}$ These results support the efficacy of assessing the exercise capacity using the $30 \mathrm{~s}$ SST. Owing to the beneficial effects achieved after a program of aerobic combined with resistance training in regard to the $30 \mathrm{~s}$ SST in patients with $\mathrm{COPD}^{36}$ and the effects achieved after a program of home-based pulmonary rehabilitation combined with lower limb resistance exercise on FTSST, ${ }^{37}$ the effects of combined exercise training in improving the exercise capacity of patients with COPD are determined. The extent of clinical supervision in exercise programs may have no impact on the intervention effects. It is also unclear whether low-intensity aerobic exercise has a positive effect and whether the extent of clinical supervision in a program of low-intensity aerobic exercise has an impact on the intervention effects. In the present study, the completed frequency of the $30 \mathrm{~s}$ SST increased (2.41 times) significantly after the intervention. The minimum clinically important difference for 1-minute SST is three times ${ }^{38}$ It may speculate that low-intensity aerobic exercise has beneficial effects on improving the exercise capacity of patients with COPD. However, further research is still needed to obtain a direct conclusion.

In the present study, the SGRQ score reflecting the quality of life significantly decreased but the quality of life improved after patients performed the Liuzijue exercise and exceeded the minimum clinically important difference of four points. ${ }^{39}$ This result was better than that of a previous study on Tai Chi among patients with COPD. ${ }^{40}$ This may be ascribed to the differences in the intervention time and exercise frequency, with 6 months of interventions six times a week in the present study and 3 months of intervention for twice a week in the former study. With regard to the extent of clinical supervision, a meta-analysis concluded that clinic-based and home-based rehabilitations exert similar effects on improving the quality of life in patients with COPD ${ }^{41}$ However, another study on home-based rehabilitation found that except for the symptom score, the SGRQ scores significantly decreased in patients with COPD. Authors reckoned that the symptoms did not improve because the patients were stable and under medicine control..$^{30}$ Another study attributed the lack of changes in the SGRQ scores to the small sample size and the Hawthorne effect. ${ }^{26}$ In addition, the increased diaphragm movement may have counteracted the reduced dyspnea during exercise. ${ }^{42}$ In conclusion, low-intensity aerobic exercise can effectively improve the quality of life of patients with COPD, and the extent of clinical supervision exerts no effect on the results.

\section{Strengths and limitations}

The main advantage of this study was the applied long-term program of family and outpatient rehabilitation to achieve a convenient and effective exercise intervention. The Liuzijue exercise was low intensity and did not require going to a 
specific location or special equipment, making this approach feasible for elderly COPD patients. In addition, the study applied two methods to assess the exercise capacity to achieve accurate results. Notably, this study also presents some limitations. First, the sample size was relatively small, while the statistical data showed a moderate effect. Second, regional differences may also be observed due to the single source of patients. Third, the influence of gender differences on the effectiveness of the intervention was not studied due to the low number of enrolled women. Therefore, a larger sample and multicenter studies are required in the future to further analyze the intervening effects. Finally, since Liuzijue exercise is a traditional Chinese fitness exercise, the efficacy may be limited with regard to the understanding and acceptability of people with different cultural backgrounds.

\section{Conclusion}

The proposed program of long-term home-based Liuzijue exercise combined with clinical guidance is safe, feasible, and effective in elderly patients with moderate to severe COPD. Therefore, this program of home-based Liuzijue exercise combined with clinical guidance should also be an alternative exercise program for elderly patients with COPD. Further studies should be conducted to confirm the effectiveness of this program and explore the optimal extent of clinical exercise supervision.

\section{Acknowledgment}

The authors would like to thank Mr Juntao Yan, Mr Longbing Wang, and Mr Chunlei Shan for their technical assistance. This work was supported by Key Laboratory of Exercise and Health Sciences of Ministry of Education, Shanghai University of Sport; the national fitness project of General Administration of Sport of China (No 2015B077, No 2017B021); the research project of the Fitness Qigong Administrative Centre of General Administration of Sport of China (No QG2017057); the "Qi Kang" young innovative talents project of Shanghai University of Traditional Chinese Medicine; and National Natural Science Foundation of China (No 81472163).

\section{Disclosure}

The authors report no conflicts of interest in this work.

\section{References}

1. Global Initiative for Chronic Obstructive Lung Disease (GOLD). Global strategy for the diagnosis, management and prevention of chronic obstructive pulmonary disease. 2018 report [cited 2017 Dec 25]. Available from: http://www.goldcopd.org. Accessed May 20, 2017.

2. Rabe KF, Watz H. Chronic obstructive pulmonary disease. Lancet. 2017;389(10082):1931-1940.
3. Maltais F, Decramer M, Casaburi R, et al. ATS/ERS Ad Hoc Committee on Limb Muscle Dysfunction in COPD. An official American Thoracic Society/European Respiratory Society statement: update on limb muscle dysfunction in chronic obstructive pulmonary disease. Am J Respir Crit Care Med. 2014;189(9):e15-e62.

4. Burtin C, Saey D, Saglam M, et al. Effectiveness of exercise training in patients with COPD: the role of muscle fatigue. Eur Respir J. 2012;40(2):338-344

5. Zwerink M, van der Palen J, van der Valk P, Brusse-Keizer M, Effing T. Relationship between daily physical activity and exercise capacity in patients with COPD. Respir Med. 2013;107(2):242-248.

6. Gimenez M, Servera E, Vergara P, Bach JR, Polu JM. Endurance training in patients with chronic obstructive pulmonary disease: a comparison of high versus moderate intensity. Arch Phys Med Rehabil. 2000;81(1):102-109.

7. Guzun R, Aguilaniu B, Wuyam B, et al. Effects of training at mild exercise intensities on quadriceps muscle energy metabolism in patients with chronic obstructive pulmonary disease. Acta Physiol (Oxf). 2012;205(2):236-246.

8. Leung RW, McKeough ZJ, Peters MJ, Alison JA. Short-form sun-style t'ai chi as an exercise training modality in people with COPD. Eur Respir J. 2013;41(5):1051-1057.

9. Xiao CM, Zhuang YC. Efficacy of Liuzijue Qigong in individuals with chronic obstructive pulmonary disease in remission. J Am Geriatr Soc. 2015;63(7):1420-1425.

10. Hong Y, Li JX, Robinson PD. Balance control, flexibility, and cardiorespiratory fitness among older Tai Chi practitioners. Br J Sports Med. 2000;34(1):29-34.

11. Wen J, Lin T, Cai Y, et al. Baduanjin exercise for type 2 diabetes mellitus: a systematic review and meta-analysis of randomized controlled trials. Evid Based Complement Alternat Med. 2017;2017:8378219.

12. Xiao CM, Zhuang YC. Effect of health Baduanjin Qigong for mild to moderate Parkinson's disease. Geriatr Gerontol Int. 2016;16(8):911-919.

13. de Araujo CL, Karloh M, Dos Reis CM, Palú M, Mayer AF. Pursedlips breathing reduces dynamic hyperinflation induced by activities of daily living test in patients with chronic obstructive pulmonary disease: a randomized cross-over study. J Rehabil Med. 2015;47(10): 957-962.

14. Chen JX. [The effects of six-character formula respiratory gymnastics on quality of life in stable patients with COPD]. Chin J Nat Med. 2009;11(6):412-414. Chinese [with English abstract].

15. Spahija J, de Marchie M, Grassino A. Effects of imposed pursed-lips breathing on respiratory mechanics and dyspnea at rest and during exercise in COPD. Chest. 2005;128(2):640-650.

16. Luo X, Zhang J, Castelberg R, et al. The effects of traditional chinese exercise in patients with chronic obstructive pulmonary disease: a meta-analysis. PLoS One. 2016;11(9):e0161564.

17. Zhang M, Xv G, Luo C, Meng D, Ji Y. Qigong Yi Jinjing promotes pulmonary function, physical activity, quality of life and emotion regulation self-efficacy in patients with chronic obstructive pulmonary disease: a pilot study. J Altern Complement Med. 2016;22(10):810-817.

18. Nasis I, Kortianou E, Vasilopoulou M, et al. Hemodynamic effects of high intensity interval training in COPD patients exhibiting exerciseinduced dynamic hyperinflation. Respir Physiol Neurobiol. 2015;217: $8-16$.

19. Vogiatzis I, Terzis G, Nanas S, et al. Skeletal muscle adaptations to interval training in patients with advanced COPD. Chest. 2005;128(6): 3838-3845.

20. Global Initiative for Chronic Obstructive Lung Disease (GOLD). Pocket guide to COPD diagnosis, management, and prevention. 2013 report [cited 2017 Dec 25]. Available from: http://www.goldcopd.org. Accessed May 21, 2017.

21. Chinese Health Qigong Association. [Chinese Health Qigong - Liu Zi Jue]. Beijing: Chinese Health Qigong Association; 2007. Chinese. Available from: http://www.chqa.org.cn/newsall.php?cid=24 . Accessed May 21, 2017. 
22. Borg GA. Psychophysical bases of perceived exertion. Med Sci Sports Exerc. 1982;14(5):377-381.

23. Miller MR, Hankinson J, Brusasco V, et al. Standardisation of spirometry. Eur Respir J. 2005;26(2):319-338.

24. ATS Committee on Proficiency Standards for Clinical Pulmonary Function Laboratories. ATS statement: guidelines for the six-minute walk test. Am J Respir Crit Care Med. 2002;166(1):111-117.

25. Ozalevli S, Ozden A, Itil O, Akkoclu A. Comparison of the sit-to-stand test with 6 min walk test in patients with chronic obstructive pulmonary disease. Respir Med. 2007;101(2):286-293.

26. Amin S, Abrazado M, Quinn M, Storer TW, Tseng CH, Cooper CB. A controlled study of community-based exercise training in patients with moderate COPD. BMC Pulm Med. 2014;14:125.

27. Güell MR, Cejudo P, Ortega F, et al. Benefits of long-term pulmonary rehabilitation maintenance program in patients with severe chronic obstructive pulmonary disease. Three-year follow-up. Am J Respir Crit Care Med. 2017;195(5):622-629.

28. Stav D, Raz M, Shpirer I. Three years of pulmonary rehabilitation: inhibit the decline in airflow obstruction, improves exercise endurance time, and body-mass index, in chronic obstructive pulmonary disease. BMC Pulm Med. 2009;9:26.

29. Daabis R, Hassan M, Zidan M. Endurance and strength training in pulmonary rehabilitation for COPD patients. Egypt J Chest Dis Tuberc. 2017;66(2):231-236.

30. de Sousa Pinto JM, Martín-Nogueras AM, Calvo-Arenillas JI, RamosGonzález J. Clinical benefits of home-based pulmonary rehabilitation in patients with chronic obstructive pulmonary disease. $J$ Cardiopulm Rehabil Prev. 2014;34(5):355-359.

31. Chan AW, Lee A, Suen LK, Tam WW. Tai chi Qigong improves lung functions and activity tolerance in COPD clients: a single blind, randomized controlled trial. Complement Ther Med. 2011;19(1):3-11.

32. Iepsen UW, Jørgensen KJ, Ringbæk T, Hansen H, Skrubbeltrang C, Lange $P$. A combination of resistance and endurance training increases leg muscle strength in COPD. Chron Respir Dis. 2015;12(2):132-145.
33. Holland AE, Spruit MA, Troosters T, et al. An official European Respiratory Society/American Thoracic Society technical standard: field walking tests in chronic respiratory disease. Eur Respir J. 2014; 44(6):1428-1446.

34. Puhan MA, Siebeling L, Zoller M, Muggensturm P, ter Riet G. Simple functional performance tests and mortality in COPD. Eur Respir J.2013;42(4): 956-963.

35. Zanini A, Aiello M, Cherubino F, et al. The one repetition maximum test and the sit-to-stand test in the assessment of a specific pulmonary rehabilitation program on peripheral muscle strength in COPD patients. Int J Chron Obstruct Pulmon Dis. 2015;10:2423-2430.

36. Covey MK, Collins EG, Reynertson SI, Dilling DF. Resistance training as a preconditioning strategy for enhancing aerobic exercise training outcomes in COPD. Respir Med. 2014;108(8):1141-1152.

37. Chen Y, Niu M, Zhang X, Qian H, Xie A, Wang X. Effects of home-based lower limb resistance training on muscle strength and functional status in stable COPD patients. J Clin Nurs. 2018;27(5-6): e1022-e1037.

38. Vaidya T, de Bisschop C, Beaumont M, et al. Is the 1-minute sit-tostand test a good tool for the evaluation of the impact of pulmonary rehabilitation? Determination of the minimal important difference in COPD. Int J Chron Obstruct Pulmon Dis. 2016;11:2609-2616.

39. Jones PW. St. George's Respiratory Questionnaire: MCID. COPD. 2005;2(1):75-79.

40. Chan AW, Lee A, Lee DT, Sit JW, Chair SY. Evaluation of the sustaining effects of Tai Chi Qigong in the sixth month in promoting psychosocial health in COPD patients: a single-blind, randomized controlled trial. ScientificWorldJournal. 2013;2013:425082.

41. Wuytack F, Devane D, Stovold E, et al. Comparison of outpatient and home-based exercise training programmes for COPD: a systematic review and meta-analysis. Respirology. 2018;23(3):272-283.

42. Bhatt SP, Luqman-Arafath TK, Gupta AK, et al. Volitional pursed lips breathing in patients with stable chronic obstructive pulmonary disease improves exercise capacity. Chron Respir Dis. 2013;10(1):5-10.
Clinical Interventions in Aging

\section{Publish your work in this journal}

Clinical Interventions in Aging is an international, peer-reviewed journal focusing on evidence-based reports on the value or lack thereof of treatments intended to prevent or delay the onset of maladaptive correlates of aging in human beings. This journal is indexed on PubMed Central, MedLine,

\section{Dovepress}

CAS, Scopus and the Elsevier Bibliographic databases. The manuscript management system is completely online and includes a very quick and fair peer-review system, which is all easy to use. Visit http://www.dovepress. com/testimonials.php to read real quotes from published authors. 